\title{
Long-wavelength effective gauge theory of the doped planar antiferromagnet
}

\author{
N. Dorey \\ Physics Department, University of Edinburgh, Mayfield Road, Edinburgh EII9 3JZ, Scotland
}

\author{
N. E. Mavromatos \\ Theory Division, CERN, CH-1211, Geneva 23, Switzerland
}

(Received 20 February 1991)

\begin{abstract}
The dynamics of holes in a planar, quantum antiferromagnet is modeled using a suitably extended $t-J$ Hamiltonian. A "slave fermion" ansatz is used to express the theory in terms of separate charge and spin variables. A gauge symmetry of the model is identified and an approximate effective Hamiltonian is given in terms of an Abelian gauge potential. The long-wavelength limit is shown to be a relativistic theory of two species of Dirac fermions, corresponding to holes on the two sublattices, coupled with opposite sign to the $\mathrm{U}(1)$ gauge field of the $C P^{1}$ nonlinear $\sigma$ model, which describes the undoped case. The usual constraint of the $C P^{1}$ model is modified to account for the presence of holes. This effective continuum theory has recently been shown to exhibit superconductivity without parity violation. The possible relevance of this model to high- $T_{c}$ superconductivity is discussed.
\end{abstract}

\section{INTRODUCTION}

The doped Heisenberg antiferromagnet in two spatial dimensions has recently been the subject of intense investigation as a model of the dynamics of strongly correlated electrons in the copper oxide layers of the high- $T_{c}$ materials $\mathrm{Y}-\mathrm{Ba}-\mathrm{Cu}-\mathrm{O}$ and $\mathrm{La}(\mathrm{Sr})-\mathrm{Cu}-\mathrm{O} .{ }^{1}$ In the case of halffilling, it is well known that the Heisenberg antiferromagnet has a long-wavelength description as a $C P^{1} \sigma$ model where the continuum fields describe fluctuations of the spins around the Néel-ordered ground state. ${ }^{2}$ The situation away from half-filling is considerably more complicated; numerical simulations indicate that the addition of holes destroys the long-range antiferromagnetic order, ${ }^{3}$ and it is not clear how the effective long-wavelength description of the system is modified.

Recently, Shankar has shown that in the limit of the doped antiferromagnet in which the electron spin $S$ is taken to be large, considerable simplifications occur. In this limit short-range antiferromagnetic order persists and the lattice is naturally partitioned into two sublattices, as shown in Fig. 1. In Ref. 4 it is shown that the amplitude for a hole to make a transition between neigh-

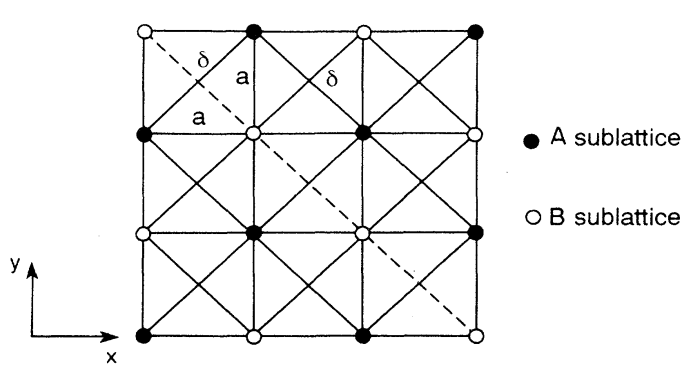

FIG. 1. Sublattice structure for hole hopping in the planar quantum antiferromagnet. boring sites on opposite sublattices is strongly suppressed. Thus, in the large- $S$ limit, holes are effectively separated into two "colors" inhabiting the two sublattices. Shankar then suggests an effective Hamiltonian in which the two colors of holes are described by Grassmann fields which couple with opposite signs to an Abelian gauge potential. The resulting long-wavelength theory consists of massless Dirac fermions minimally coupled to the $\mathrm{U}(1)$ gauge field of the $C P^{1} \sigma$ model. This result has been demonstrated for arbitrary doping concentration for the case of a spin chain ${ }^{4}$ (i.e., one spatial dimension), and the derivation can be generalized to the two-dimensional case in the limit of light doping, ${ }^{5}$ where the resulting effective action is given by

$$
S_{\mathrm{eff}}=\int d^{3} x\left[\frac{1}{\gamma}\left|\left(\partial_{\mu}+g i a_{\mu}\right) z\right|^{2}+\bar{\Psi}_{a}\left(i \not \partial-g d \tau_{3}\right) \Psi_{a}\right),
$$

where $\bar{z} z=1$ and the matrix $\tau_{3}$ acts in the two-component color space and is given by $\operatorname{diag}(1,-1)$. The "flavor" in$\operatorname{dex} a=1,2$ arises because the Grassmann fields have been assembled as staggered lattice fermions. The above action has an "accidental" relativistic invariance and is written in natural units of which $\hbar=v=1$, where the characteristic velocity $v$ is identified with the Fermi velocity for holes, $v_{F}$.

The action (1) has recently been proposed as a model of planar superconductivity. ${ }^{5,6}$ The gauge field provides an attractive potential between fermions of opposite color, and an approximate treatment of the Schwinger-Dyson equation for the fermion propagator indicates that a parity-conserving mass gap $\Delta \bar{\psi} \tau_{3} \psi$ is dynamically generated because of this interaction. In the massive phase, the $U(1)$ of electric charge is spontaneously broken, leading to the superconductivity and the Meissner effect. In classical two-dimensional statistical mechanics, spontane- 
ous breaking of a continuous symmetry at nonzero temperature is forbidden by the Mermin-Wagner theorem, ${ }^{7}$ and this result is also thought to apply to finitetemperature QFT in $(2+1)$ dimensions, ${ }^{8}$ by an extension of the arguments of Ref. 9. However, it can be shown that the symmetry in question is broken without the appearance of a local order parameter, and so a finite transition temperature is not in contradiction with the theorem. Among many other attractive features, it appears that this model exhibits a mass-gap-to-criticaltemperature ratio much larger than that given by conventional BCS theory, in accord with experimental results for the planar high- $T_{c}$ materials. ${ }^{10}$

Given the above, it is of considerable interest to investigate further the possible microscopic origin of such an effective continuum theory. Of course, the assumptions underlying Shankar's model are valid only in the large- $S$ limit. However, the philosophy adopted in this paper will be to assume that certain qualitative features of the large- $S$ limit may be safely abstracted to the realistic case $S=\frac{1}{2}$. In particular, it will be assumed that intersublattice hopping is still suppressed and therefore that the integrity of the two colors of holes is preserved, even for $S=\frac{1}{2}$. This assumption can be incorporated in a suitably extended $t-J$ model which describes the hole hopping within each sublattice as well as the Heisenberg interactions between spins. Although this model is formulated in terms of electron creation and annihilation operators, it will be possible to rewrite the Hamiltonian in terms of separate degrees of freedom describing the spins and holes using a variant of the "slave-boson" ansatz. These new variables may then be identified with those occurring in Shankar's formulation. The aim of this work is to investigate the relation between Shankar's model and more conventional models of strongly correlated electronic systems and, at the same time, clarify the assumptions underlying the continuum model of planar superconductivity presented in Refs. 5 and 6 . The main result of this paper is that an effective action closely related to (1) arises naturally as the long-wavelength limit of the $t-J$ model.

The paper is arranged as follows: Section I describes properties of the large- $S$ antiferromagnet and consists mainly of a review of the relevant parts of Ref. 4. Section II describes the $t-J$ model and the "slave-fermion" ansatz, and in Sec. III the effective long-wavelength theory is derived.

\section{LARGE-S ANTIFERROMAGNET}

The two-dimensional, undoped, spin- $S$, quantum Heisenberg antiferromagnet is described by the Hamiltonian

$$
H=J \sum_{i, j} \mathbf{s}_{i} \cdot \mathbf{s}_{j}
$$

where $i$ and $j$ are nearest-neighbor sites on a square lattice. The spin operators $s_{i}=\left(s^{a}\right)_{i}$ obey independent $O(3)$ commutation relations at each site:

$$
\left[s_{i}^{a}, s_{j}^{b}\right]=i \epsilon^{a b c} s_{i}^{c} \delta_{i j} .
$$

At zero temperature the classical ground state of the an- tiferromagnet is just the Néel-ordered state defined by $\mathrm{s}_{i}=S(-1)^{I} \widehat{\mathrm{z}}$, where $(-1)^{I}$, with $I=i_{x}+i_{y}$, is the parity of the site $i$ and $\widehat{\mathbf{z}}$ is a unit vector.

Following Haldane, ${ }^{2}$ the Hilbert space of the system is conveniently described in terms of a basis of coherent states $|\boldsymbol{\Omega}(i)\rangle$, where $\boldsymbol{\Omega}(i)$ is a unit vector with angular coordinates $\left(\theta_{i}, \phi_{i}\right)$ and

$$
\begin{aligned}
\langle\boldsymbol{\Omega}(i) \mid \mathbf{\Omega}(j)\rangle= & {\left[\cos \left[\frac{\theta_{i}}{2}\right] \cos \left[\frac{\theta_{j}}{2}\right]+\exp i\left(\phi_{i}-\phi_{j}\right)\right.} \\
& \left.\times \sin \left(\frac{\theta_{i}}{2}\right) \sin \left[\frac{\theta_{j}}{2}\right]\right]^{2 S}, \\
\left\langle\boldsymbol{\Omega}(i)\left|\mathbf{s}_{i}\right| \mathbf{\Omega}(i)\right\rangle= & S \boldsymbol{\Omega}(i) .
\end{aligned}
$$

From (4) it can be seen that the overlap of neighboring coherent states decays rapidly with angular separation in the large-S limit. This relation can be written as

$$
\langle\mathbf{\Omega}(i) \mid \mathbf{\Omega}(j)\rangle=\exp (\text { is } \mathbf{A}(\mathbf{\Omega}) \cdot \Delta \mathbf{\Omega}),
$$

where $\Delta \boldsymbol{\Omega}=\boldsymbol{\Omega}(i)-\boldsymbol{\Omega}(j)$ and $\mathbf{A}(\boldsymbol{\Omega})$ is the potential describing a unit monopole at the origin:

$$
\boldsymbol{\nabla}_{\boldsymbol{\Omega}} \times \mathbf{A}(\boldsymbol{\Omega})=\boldsymbol{\Omega} .
$$

As usual, the potential $\mathbf{A}$ is not uniquely defined by (7), and in particular, a gauge can be chosen in which $\mathbf{A}(\boldsymbol{\Omega})=\mathbf{A}(-\boldsymbol{\Omega})^{4}$

In the large-S limit, the partition function of the system may be written as a path integral over the variables $\boldsymbol{\Omega}$. In order to take a continuum limit, it is necessary to define an order parameter field $\mathbf{n}(i)$ that varies smoothly in space. As the system has a Néel-ordered ground state, the field $\boldsymbol{\Omega}(i)$ alternates in direction from site to site; so $\mathbf{n}(i)$ is defined as

$$
\mathbf{n}(i)=(-1)^{I} \boldsymbol{\Omega}(i)+O(a),
$$

where $a$ is the lattice spacing. In the long-wavelength limit, the effective action may be written in terms of the continuum field $\mathbf{n}(\mathbf{x}, t)$ as an $O(3)$ nonlinear $\sigma$ model:

$$
S=\int d^{3} x \frac{1}{\gamma}\left(\partial_{\mu} n\right)\left(\partial^{\mu} \mathbf{n}\right)
$$

The $\sigma$-model action may be rewritten in terms of $C P^{1}$ variables in the usual way by making the substitution $\mathbf{n}=\bar{z} \sigma z$, with $\bar{z} z=1$. This gives

$$
S=\int d^{3} x \frac{1}{\gamma}\left|\left(\partial_{\mu}+i a_{\mu}\right) z\right|^{2},
$$

where the $\mathrm{U}(1)$ gauge field $a_{\mu}$ is determined by its equation of motion: $a_{\mu}=i \bar{z} \partial_{\mu} z$. The above result is valid only at half-filling, but the results of this investigation will suggest that the introduction of holes is accurately modeled by coupling the $C P^{1} \sigma$ model to relativistic Dirac fermions via the gauge field $a_{\mu}$.

In Ref. 4 a formalism for describing the model away from half-filling is developed, introducing fermionic operators $\psi^{\dagger}$ and $\psi$ to describe the creation and annihilation of holes. A key point of Shankar's analysis is that the amplitude for a hole to hop from site $i$ to site $j$ is the 
product of the overlap of the spatial and spin wave functions describing the initial and final states. The spin part is proportional to the factor

$$
\langle\boldsymbol{\Omega}(i) \mid \boldsymbol{\Omega}(j)\rangle=\exp (i S \mathbf{A}(\boldsymbol{\Omega}) \cdot \Delta \boldsymbol{\Omega}) .
$$

If $i$ and $j$ are neighboring sites in opposite sublattices, then the coherent states $|\boldsymbol{\Omega}(i)\rangle$ and $|\Omega(j)\rangle$ are almost orthogonal by virtue of (4). Hence, as stated above, intersublattice hopping is highly supressed in the large- $S$ limit. If sites $i$ and $j$ live on the same sublattice, then the spin overlap may be written in terms of the $\sigma$-model order parameter using (6):

$$
\langle\mathbf{\Omega}(i) \mid \mathbf{\Omega}(j)\rangle=\exp \left(i S(-1)^{I} \mathbf{A} \cdot \Delta \mathbf{n}\right) .
$$

As the field $\mathbf{n}$ is only slowly varying in space, this expression has a smooth continuum limit for each sublattice as $a \rightarrow 0$. This suggests that the appropriate Hamiltonian to describe hole hopping consists of two species of Grassmann fields coupled to a gauge field with opposite sign.

This implies a short-range attraction between holes on opposite sublattices which may be attributed to a destructive interference of Berry-phase factors of spins at neighboring lattice sites. ${ }^{4}$ There is also a tendency of holes to reside on NN sites, which can be understood from simple energetics arguments. The creation of two holes in the Néel-ordered ground state requires the breaking of eight antiferromagnetic bonds which increases the energy of the system by $8 J S^{2}$ unless the holes are nearest neighbors, in which case one less bond is broken and the energy cost is only $7 J S^{2}$.

\section{III. $t$-J MODEL}

The $t-J$ Hamiltonian describes the dynamics of electrons located at sites $i=\left(i_{x}, i_{y}\right)$ of a square twodimensional lattice and is written in terms of electron creation and annihilation operators $C_{i, \alpha}^{\dagger}$ and $C_{i, \alpha}$ obeying the usual anticommutation relations

$$
\begin{aligned}
& \left\{C_{i, \alpha}^{\dagger}, C_{j, \beta}\right\}_{+}=\delta_{i j} \delta_{\alpha \beta}, \quad\left\{C_{i, \alpha}^{\dagger}, C_{j, \beta}^{\dagger}\right\}_{+}=0, \\
& \left\{C_{i, \alpha}, C_{j, \beta}\right\}_{+}=0 .
\end{aligned}
$$

The index $\alpha=1,2$ labels the spin state of the electron, and the convention of summation over repetitions of this index is understood throughout. The full $t-J$ Hamiltonian, including both nearest-neighbor (NN) and next-nearestneighbor (NNN) interactions, is given by

$$
\begin{aligned}
H= & H_{t}+H_{t^{\prime}}+H_{J}+H_{J^{\prime}}+H_{\mu} \\
= & -t \sum_{[i j]} C_{i, \alpha}^{\dagger} C_{j, \alpha}-t^{\prime} \sum_{\langle i j\rangle} C_{i, \alpha}^{\dagger} C_{j, \alpha}+J \sum_{[i j]} T_{i, \alpha \beta} T_{j, \beta \alpha} \\
& +J^{\prime} \sum_{\langle i j\rangle} T_{i, \alpha \beta} T_{j, \beta \alpha}-\mu \sum_{i} C_{i, \alpha}^{\dagger} C_{i, \alpha},
\end{aligned}
$$

where $[i j]$ indicates that $i$ and $j$ are $\mathrm{NN}$ sites and $\langle i j\rangle$ that they are NNN. $T_{i, \alpha \beta}$ are the spin $\mathrm{SU}(2)$ generators and are given in terms of the electron operators by $T_{i, \alpha \beta}=C_{i, \alpha}^{\dagger} C_{i, \beta}$. The terms $H_{J}$ and $H_{J^{\prime}}$ represent the Heisenberg interaction between electron spins at NN and NNN sites. In physical models the strength of this in- teraction may decrease rapidly with separation so that typically $J^{\prime} \ll<J$, and later in the calculation the case $J^{\prime} \rightarrow 0$ will be considered explicitly. $H_{\mu}$ is a chemical potential term which is introduced to enforce the local constraint $\left\langle C_{i, \alpha}^{\dagger} C_{i, \alpha}\right\rangle=1-\delta$, where $\delta$ is the doping concentration.

The terms $H_{t}$ and $H_{t^{\prime}}$ express the contribution of processes in which an electron (or, equivalently, a hole) hops between NN and NNN sites, respectively, and the constants $t$ and $t^{\prime}$ are proportional to the relevant overlap integrals. The assumption of no intersublattice hopping will be implemented by setting $t=0$ and retaining only the NNN hopping term. It will often be convenient to rewrite the NNN hopping term describing NN hopping within each sublattice:

$$
H_{t^{\prime}}=-t^{\prime} \sum_{i \in A, \delta} C_{i, \alpha}^{\dagger} C_{i+\delta, \alpha}+(A \rightarrow B),
$$

where $\delta$ is the NNN separation vector (see Fig. 1).

In order to rewrite the $t-J$ Hamiltonian in a more tractable form, several authors have utilized a slave-boson ansatz (see, for example, Ref. 11, and references therein) in which the electron operator $C^{\dagger}$ is written as a product of a fermionic field carrying a spin index and a spin-singlet slave boson. Here the aim is to make contact with Shankar's formulation in which the Grassmann fields representing the holes do not carry a spin index. Consequently, an alternative, slave-fermion ansatz for the electron operator is introduced:

$$
C_{i, \alpha}^{\dagger}=\psi_{i} z_{i, \alpha}^{\dagger},
$$

where $\psi$ and $z$ carry fermionic and bosonic statistics, respectively:

$$
\begin{aligned}
& \left\{\psi_{i}^{\dagger}, \psi_{j}\right\}_{+}=\delta_{i j}, \quad\left\{\psi_{i}^{\dagger}, \psi_{j}^{\dagger}\right\}_{+}=0, \quad\left\{\psi_{i}, \psi_{j}\right\}_{+}=0, \\
& {\left[z_{i, \alpha}^{\dagger}, z_{j, \beta}\right]=\delta_{i j} \delta_{\alpha \beta}, \quad\left[z_{i, \alpha}^{\dagger}, z_{j, \beta}^{\dagger}\right]=0,\left[z_{i, \alpha}, z_{j, \beta}\right]=0 .}
\end{aligned}
$$

A similar ansatz has been used, in a different context, in Ref. 13 to study normal-state properties of the antiferromagnet. However, these authors ignore the Heisenberg interaction terms, which turn out to be important for a study of the superconducting properties of the model. An important feature of the ansatz (16) is that the resulting effective Hamiltonian is invariant under local phase rotations that leave the original electron operators invariant:

$$
\psi_{i} \rightarrow \exp \left(i g \theta_{i}\right) \psi_{i}, \quad z_{i} \rightarrow \exp \left(i g \theta_{i}\right) z_{i} .
$$

Unlike the formalism of Baskaran and Anderson, ${ }^{14}$ the $\mathrm{U}(1)$ gauge symmetry is maintained in this approach, even away from half-filling. A similar conclusion is reached in the slave-boson approach of Ref. 15.

The fields $\psi_{i}$ and $z_{i, \alpha}$ are not independent; using (17) and (18) gives the following constraint:

$$
\frac{1}{2} z_{i, \alpha}^{\dagger} z_{i, \alpha}+\psi_{i}^{\dagger} \psi_{i}=1 \text {. }
$$

Interpreting $\psi_{i}, \psi_{i}^{\dagger}$ as annihilation and creation operators for holes, this operator relation simply expresses the fact that the sum of number of electrons and number of holes at any given site is two, one for each spin state. Express- 
ing the Hamiltonian (2) in terms of the new variables,

$$
\begin{aligned}
H= & -t^{\prime} \sum_{\langle i j\rangle} \psi_{i} \psi_{j}^{\dagger} z_{i, \alpha}^{\dagger} z_{j, \alpha}+J \sum_{[i, j]} \psi_{i} \psi_{i}^{\dagger} \psi_{j} \psi_{j}^{\dagger} z_{i, \alpha}^{\dagger} z_{i, \beta} z_{j, \beta^{\dagger}}^{\dagger} z_{j, \alpha} \\
& +\left(J \rightarrow J^{\prime},[i j] \rightarrow\langle i j\rangle\right)-\mu \sum_{i} z_{i, \alpha}^{\dagger} z_{i, \alpha} \psi_{i} \psi_{i}^{\dagger}
\end{aligned}
$$

Using the constraint (20) and the anticommutation relations for $\psi_{i}$, the chemical potential term becomes

$$
H_{\mu}=\mu \sum_{i} \psi_{i}^{\dagger} \psi_{i}+\text { const }
$$

The Heisenberg interaction terms $H_{J}$ and $H_{J^{\prime}}$ can be simplified by splitting the fermionic products $\psi_{i} \psi_{i}^{\dagger}$ into a vacuum expectation value and a normal-ordered part of the usual way:

$$
\psi_{i} \psi_{i}^{\dagger}=\left\langle\psi_{i} \psi_{i}^{\dagger}\right\rangle+: \psi_{i} \psi_{i}^{\dagger}
$$

After the usual rescalings, the normal ordered part $: \psi_{i} \psi_{i}^{\dagger}$ : has scaling dimension two and corrections such as $: \psi_{i} \psi_{i}^{\dagger}: z_{i, \alpha}^{\dagger} z_{i, \beta} z_{j, \beta}^{\dagger} z_{j, \alpha}$ will have dimension four in the continuum limit and are thus irrelevant operators by power counting. ${ }^{4}$ Keeping only the marginal contribution yields

$$
H_{J}=J \eta^{2} \sum_{[i, j]} z_{i, \alpha}^{\dagger} z_{i, \beta^{\prime}} z_{j, \beta}^{\dagger} z_{j, \alpha}
$$

with $\eta=\left\langle\psi_{i} \psi_{i}^{\dagger}\right\rangle$ and similarly for $H_{J^{\prime}}$.

The hopping term $H_{t^{\prime}}$ and the leading Heisenberg terms in $H_{J}$ and $H_{J^{\prime}}$ are quartic in the fields and may be linearized by introducing an auxiliary link variable $\Delta_{i j}$. This is done by subtracting the following Gaussian term from the Hamiltonian. In the path-integral formalism which will be adopted below this changes the normalization of the partition function by a trivial multiplicative constant:

$$
H_{\Delta}=\sum_{[i, j],\langle i j\rangle}\left|\Delta_{i j}-b_{i j} z_{i, \alpha}^{\dagger} z_{j, \alpha}-c_{i j} \psi_{i}^{\dagger} \psi_{j}\right|^{2}
$$

The constants $b_{i j}$ and $c_{i j}$ are chosen to be

$$
\begin{aligned}
& b_{[i j]}=\left(J \eta^{2}\right)^{1 / 2}, \quad c_{[i j]}=0, \\
& b_{\langle i j\rangle}=\left(J^{\prime} \eta^{2}\right)^{1 / 2}, c_{\langle i j\rangle}=-\frac{t^{\prime}}{\left(J^{\prime} \eta^{2}\right)^{1 / 2}}, \\
& 2 b_{i i}=c_{i i}=1,
\end{aligned}
$$

so that the quartic terms are eliminated from the Hamiltonian and classically $\Delta_{i i}=1$. The complete Hamiltonian reads

$$
H=H_{\Delta z}+H_{\Delta \psi}+H_{\Delta^{2}}+H_{4 F}+H_{\mu} .
$$
by

The four-fermion contact interaction term $H_{4 F}$ is given

$$
\begin{aligned}
H_{4 F} & =-\sum_{\langle i j\rangle}\left(\frac{t^{\prime 2}}{J^{\prime} \eta^{2}}\right] \psi_{i}^{\dagger} \psi_{j} \psi_{i} \psi_{j}^{\dagger} \\
& =\frac{t^{\prime 2}}{2 J^{\prime} \eta^{2}} \sum_{\langle i j\rangle}\left(\psi_{i}^{\dagger} \psi_{i}-\psi_{j}^{\dagger} \psi_{j}\right)^{2} .
\end{aligned}
$$

Although such terms are perturbatively irrelevant by naive power counting in $(2+1)$ spacetime dimensions, some care is required. Certain relativistic four-fermion operators become relevant in the large- $N$ expansion ${ }^{12}$ (where $N$ is the number of fermion flavors). In fact, this is true only for attractive interactions and $H_{4 F}$ is repulsive and so remains irrelevant. However, microscopic models which yield attractive relativistic four-fermion interactions in the long-wavelength limit can easily be constructed. $^{5}$ In that case the four-fermion operator corresponds to the difference of the asymmetry of NNN hopping in the two sublattices and contributes to the dynamical generation of a parity-conserving mass gap. The terms involving $\Delta_{i j}$ are given by

$$
\begin{aligned}
H_{\Delta z}= & \sum_{[i j]}\left(J \eta^{2}\right)^{1 / 2}\left(z_{i, \alpha}^{\dagger} \Delta_{i j} z_{j, \alpha}\right)+\text { H.c. } \\
& +\left(J \rightarrow J^{\prime},[i j] \rightarrow\langle i j\rangle\right), \\
H_{\Delta \psi}= & \sum_{\langle i j\rangle}\left[\frac{-t^{\prime}}{\left(J^{\prime} \eta^{2}\right)^{1 / 2}}\right] \psi_{i}^{\dagger} \Delta_{i j} \psi_{j}+\text { H.c. }, \\
H_{\Delta^{2}}= & -\sum_{[i j],\langle i j\rangle}\left|\Delta_{i j}\right|^{2} .
\end{aligned}
$$

\section{LONG-WAVELENGTH EFFECTIVE ACTION}

The model is now expressed in terms of bilocal fields $\Delta_{i j}$, which obey a gauge transformation law:

$$
\Delta_{i j} \rightarrow \exp \left[-i g\left(\theta_{j}-\theta_{i}\right)\right] \Delta_{i j} .
$$

As $\Delta_{i j}$ is a gauge-variant quantity, its expectation must vanish by Elitzur's theorem. Following the approach of Baskaran and Anderson ${ }^{14}$ only the phase fluctuations of the auxiliary field $\Delta_{i j}$ around its mean value are considered and the approximation

$$
\begin{aligned}
& \Delta_{[i j]} \simeq \Delta \exp \left(i g a_{i j}\right), \\
& \Delta_{\langle i j\rangle} \simeq \Delta^{\prime} \exp \left(i g a_{i j}\right)
\end{aligned}
$$

is now adopted. The term $H_{\Delta^{2}}$ depends only on the moduli $\Delta$ and $\Delta^{\prime}$ and does not contribute to the dynamics. The remaining terms $H_{\Delta z}$ and $H_{\Delta \psi}$ now take on a form reminiscent of a $U(1)$ lattice gauge theory in $(2+1)$ dimensional spacetime. Rewriting $H_{\Delta \psi}$ in terms of the two sublattices, as in (15), yields

$$
\begin{aligned}
H_{\Delta \psi}= & -G \sum_{i \in A, \delta} \psi_{i}^{\dagger} \exp [\operatorname{ig} \widetilde{\mathbf{a}}(i) \cdot \delta] \psi_{i+\delta} \\
& + \text { H.c. }+(A \rightarrow B)
\end{aligned}
$$

where $G=\Delta^{\prime} t^{\prime} /\left(J^{\prime} \eta^{2}\right)^{1 / 2}, \widetilde{\mathbf{a}}(i) \cdot \delta=a_{\langle i j\rangle}$, and $\delta$ is the vector separating sites $i$ and $j$. The hopping term is responsible for the transport of a hole between two neighboring sites in the same sublattice. According to Shankar's analysis, the amplitude for this process is proportional to $\langle\boldsymbol{\Omega}(i) \mid \boldsymbol{\Omega}(j)\rangle$ in the large-S limit, and comparing (6) and (35), this suggests the identification

$$
g \widetilde{\mathbf{a}}(i) \cdot \boldsymbol{\delta} \simeq(-1)^{I} S \mathbf{A}(\boldsymbol{\Omega}) \cdot \Delta \mathbf{n}(i) .
$$

In order to take a continuum limit, it is necessary to 
define a smoothly varying gauge field a $(i)$. Assuming that the identification (36) remains qualitatively correct in the realistic case $S=\frac{1}{2}$, this can be done by setting $\mathbf{a}(i)=(-1)^{I} \widetilde{\mathbf{a}}(i)$. This gives

$$
\begin{aligned}
H_{\Delta \psi}= & -G \sum_{i \in A, \delta} \psi_{i}^{\dagger} \exp [\operatorname{ig} \mathbf{a}(i) \cdot \delta] \psi_{i+\delta} \\
& + \text { H.c. }+(A \rightarrow B, g \rightarrow-g) .
\end{aligned}
$$

This is nothing other than the hole-hopping term of Shankar's model.

The hole-hopping Hamiltonian (37) describes two species of Grassmann fields interacting with a $U(1)$ gauge potential. Employing the approach of Ref. 16, the background gauge-field configuration will be initially fixed to one in which the flux per sublattice placquette is equal to $\pi$, or in other words, the product of group elements around a sublattice placquette is equal to -1 . Fluctuations around this background gauge configuration will be reintroduced once the continuum limit has been taken. In order to derive a continuum theory of Dirac spinors, the Grassmann fields on each sublattice can be assembled as Kogut-Susskind lattice fermions. ${ }^{17}$ Representing an arbitrary site in sublattice $A$ as $i=n_{x} \delta_{x}+n_{y} \delta_{y}$, where $\delta_{x}$ and $\delta_{y}$ are orthogonal unit vectors of sublattice $A$ (see Fig. 1), spinor components are defined according to the parity of the integers $n_{x}$ and $n_{y}$ as $\psi^{1}=$ (even, even), $\psi^{2}=$ (even, odd), $\psi^{3}=$ (odd, even), and $\psi^{4}=$ (odd, odd).

Including the chemical potential term $H_{\mu}$, the hopping Hamiltonian for sublattice $A$ is given in momentum space by

$$
H_{A}=-G \int d^{2} k \psi^{\dagger i}(k)[M(k)]_{i j} \psi(k)^{j},
$$

where the $k$ integration is restricted to the appropriate Brillouin zone and the $4 \times 4$ matrix $M(k)$ is given by

$$
M(k)=\left(\begin{array}{cccc}
-M & S_{y} & -i S_{x} & 0 \\
S_{y} & -M & 0 & i S_{x} \\
i S_{x} & 0 & -M & S_{y} \\
0 & -i S_{x} & S_{y} & -M
\end{array}\right) .
$$

Here $M=\mu / G$ and $S_{i}=-\sin \left(k_{i} / G\right)$, with $k_{i}=\mathbf{k} \cdot \delta_{i}$ and $i=x, y$. The Hamiltonian (38) can be recast in block diagonal form by a suitable change of basis in spinor space:

$$
H_{A}=-G \int d^{2} k \chi_{a}^{\dagger}[\tilde{M}(k)]_{a b} \chi_{b},
$$

where $\chi_{a}, a=1,2$ is now a vector of two-component spinors given by $\chi_{1}=1 / \sqrt{2}\left(\psi_{1}+\psi_{4}, \psi_{2}+\psi_{3}\right)$ and $\chi_{2}$ $=1 / \sqrt{2}\left(\psi_{2}-\psi_{3}, \psi_{1}-\psi_{4}\right)$ and

$$
\tilde{M}(k)=\left(\begin{array}{cc}
S_{x} \sigma_{x}+S_{y} \sigma_{y}+M \mathcal{J} & 0 \\
0 & S_{x} \sigma_{x}+S_{y} \sigma_{y}+M \mathcal{J}
\end{array}\right),
$$

where $\sigma_{i}$ are the usual Pauli matrices and $\mathscr{I}$ is he $2 \times 2$ unit matrix.

The energy spectrum of the hopping Hamiltonian is obtained by solving the eigenvalue equation of the matrix $\widetilde{M}(k)$ :

$$
E(k)=\mu \pm G\left[S_{x}^{2}+S_{y}^{2}\right]^{1 / 2}=\mu \pm \epsilon(k) .
$$

The long-wavelength continuum limit is to be taken by expanding the Hamiltonian in powers of momenta around the Fermi surface and retaining only linear terms. In particular, the Fermi surface is defined by the relation $\epsilon(k)=\mu$ and the momenta may be split as ' $\mathbf{k}=\mathbf{k}_{F}+\mathbf{k}^{\prime}$, where $\mathbf{k}_{F}$ is a particular expansion point on the Fermi surface. New spinors $\hat{\chi}_{a}$ and $\hat{\chi}_{a}^{\dagger}$ are defined as zero modes of the matrix $\tilde{M}\left(k_{F}\right)$ and the Hamiltonian can then be written, to leading order of $\mathbf{k}^{\prime}$, as

$$
H_{A}=-G \int d^{2} k^{\prime} \hat{\chi}_{a}^{\dagger}\left(C_{i}^{F} \sigma_{i} k_{i}^{\prime}\right) \hat{\chi}_{a},
$$

where $C_{i}^{F}=\cos \left(k_{F i} / G\right)$ and the summation over $i=x, y$ is implied. In the one-dimensional case the dependence of this result on the Fermi momentum $k_{F}$ could be absorbed by simply rescaling the coupling $G$ by a factor $C_{1}^{F}$. In this case there is also dependence on an arbitrary choice of direction in the plane and an additional rescaling of momentum component $k_{2}^{F}$ by a factor $C_{2}^{F} / C_{1}^{F}$ is required to absorb this. As mentioned above, in physical systems $J^{\prime} \ll J$, and it is now convenient to consider the simplified case $J^{\prime} \rightarrow 0$. In this limit $G \rightarrow \infty$ and $C_{i}^{F} \rightarrow 1$. Hence all dependence on the expansion point chosen disappears and the Hamiltonian (43) takes on the standard Dirac form. Taking the Fourier transform and restoring the fluctuations of the gauge field around its background value gives

$$
H_{A}=-G \int d^{2} x \hat{\chi}_{a}^{\dagger} \sigma^{i}\left(\partial_{i}-g a_{i}\right) \hat{\chi}_{a} .
$$

Hence the long-wavelength limit of the hopping term for sublattice $A$ describes a theory of two flavors (corresponding to the index $a=1,2$ ) of two-component Dirac fermions minimally coupled to a $U(1)$ gauge field with coupling $g$. The whole procedure is easily repeated for the Grassmann fields inhabiting sublattice $B$; this yields two more flavors of Dirac fermions coupled to the gauge field with coupling $-g$. Combining the spinors from the two sublattices in a two-component "vector" $\Psi$, the complete Hamiltonian for the fermionic sector of the model becomes

$$
H_{\Psi}=H_{A}+H_{B}=\int d^{2} x \Psi_{a}^{\dagger} \sigma^{i}\left(\partial_{i}-g \tau_{3} a_{i}\right) \Psi_{a},
$$

where the matrix $\tau_{3}$ is given by $\operatorname{diag}(1,-1)$ acting on the internal index of $\Psi$, which labels the sublattices $A$ and $B$.

Returning to the bosonic sector of the model, in the limit $J^{\prime} \rightarrow 0$, the Heisenberg terms become

$H_{\Delta z}=\sum_{i, \mu}\left(J \eta^{2}\right)^{1 / 2} z_{i, \alpha}^{\dagger} \Delta \exp [i g \mathbf{b}(i) \cdot \mu] z_{j, \alpha}+$ H.c. ,

where $\mathbf{b}(i) \cdot \boldsymbol{\mu}=a_{[i j]}$ and $\boldsymbol{\mu}$ is the vector separating $\mathrm{NN}$ sites $i$ and $j$. This is the usual coupling of a complex scalar doublet to a $U(1)$ lattice gauge field. Taking a naive continuum limit $|\mu| \rightarrow 0$ gives

$$
H_{\Delta z}=\int d^{2} x\left(J \eta^{2}\right)^{1 / 2} \Delta\left|\left(\partial_{i}-i g b_{i}\right) z\right|^{2},
$$

where $i=x, y$ and $z=\left(z_{1}, z_{2}\right)$. This differs from the Hamiltonian of the $C P^{1} \sigma$ model only in the constraint, which is no longer simply $\bar{z} z=1$, but now also depends on the 
fermion fields. In principle, the gauge fields $\mathbf{a}(\mathbf{x})$ and $\mathbf{b}(\mathbf{x})$ are independent, and this introduces an extra $U(1)$ gauge symmetry of the effective Hamiltonian, which is actually broken by the terms of order $J^{\prime}$, which have been dropped. This redundant degree of freedom may be fixed by making the gauge choice $\mathbf{a}(\mathbf{x})=\mathbf{b}(\mathbf{x})$, which is consistent with the gauge invariance (19) of the underlying model.

By introducing a continuous Matsubara time $\tau \in(0, \beta)$, where $\beta^{-1}=k_{B} T$, the partition function of the system may be written as a Euclidean path integral over spacetime fields $\Psi(\mathbf{x}, \tau)$ and $z(\mathbf{x}, \tau)$ :

$$
\begin{aligned}
Z=\int & \mathscr{D} \bar{z} \mathscr{D} z \mathscr{D} \Psi^{\dagger} \mathscr{D} \Psi \mathscr{D} a_{i} \\
& \times \exp \left(-S_{0}+\int_{0}^{\beta} d \tau\left(H_{\Delta z}+H_{\Psi}\right)\right],
\end{aligned}
$$

where

$$
S_{0}=\int_{0}^{\beta} d \tau \int d^{2} x \frac{1}{v_{F}} \Psi_{a}^{\dagger} \gamma_{0} \partial_{\tau} \Psi_{a}+\frac{1}{v_{S}^{2}} \partial_{\tau} \bar{z} \partial_{\tau} z
$$

The characteristic velocities $v_{F}$ and $v_{S}$ are the Fermi velocity for holes and spin-wave velocity, respectively. For simplicity, we assume these are of the same order of magnitude and work in natural units, setting $v_{F}=v_{S}=1$, but one could also consider a more general model in which the ratio $v_{F} / v_{S}$ is retained as an adjustable parameter and the relativistic form of (48) is modified. Finally, the effective long-wavelength action of the system coincides with that of a fully gauge-invariant theory $(2+1)$ dimensional spacetime in which the temporal gauge condition $a_{0}=0$ has been chosen. Writing $d^{3} x=d \tau d^{2} x$,

$$
S_{\mathrm{eff}}=\int d^{2} x\left[\frac{1}{\gamma}\left|\left(\partial_{\mu}+i g a_{\mu}\right) z\right|^{2}+\bar{\Psi}_{a}\left(i \not-g d \tau_{3}\right) \Psi_{a}\right],
$$

where $\gamma=\left(J \eta^{2} \Delta^{2}\right)^{-1 / 2}$ and the Dirac algebra is given by $\gamma_{\mu}=i\left(\sigma_{z}, \sigma_{x}, \sigma_{y}\right)$. The freedom to redefine path-integral variables has been used to express the action in terms of $\bar{\Psi}=\Psi^{\dagger} \gamma_{0}$. In the continuum limit the constraint $(20)$ becomes

$$
\bar{z} z+\frac{2}{G^{2}} \Psi_{a}^{\dagger} \Psi_{a}=\text { const },
$$

and this may be implemented in the path integral by introducing a Lagrange multiplier field in the usual way. As claimed, this effective continuum action coincides with that of the model considered in Refs. 5 and 6.

\section{CONCLUSION}

The analysis of the $t-J$ model presented here indicates that the effect of adding holes to the quantum antiferromagnet is correctly described in the long-wavelength limit by coupling two species of Dirac fermions, with opposite sign, to the $\mathrm{U}(1)$ gauge field of the $C P^{1} \sigma$ model that describes the half-filled case. The resulting continuum model immediately suggests a mechanism for super- conductivity, namely, hole-pair condensation due to the gauge attraction between holes on opposite sublattices. This scenario has been investigated quantitatively in Refs. 5 and 6, using an expansion in $1 / N$, where $N$ is the number of fermion flavors, and for completeness these results are briefly described here.

The leading-order contribution to the two-point function $\left\langle a_{\mu} a_{v}\right\rangle$ involves an infinite sum of Feynman graphs, and although the gauge field in (50) has no kinetic term, this sum leads to a long-range Coulombic $(1 / R)$ static potential between charges. The quantum-mechanical analysis of Ref. 6 shows that this attraction is strong enough to cause fermions of opposite charge to condense in $s$-wave Cooper-like pairs. As stated above, the existence of a short-range attraction between holes can be understood from the overlap of Berry-phase factors between spins at NN sites. The results of the $1 / N$ expansion indicate that polarization effects in the gas of holes promote this short-range interaction to a long-range one, capable of binding holes together in bosonic pairs. As in BCS theory, these pairs condense at low temperature, giving rise to a superconducting ground state. It would be interesting to try to understand this phenomenon more directly in terms of the electron operators of the $t-J$ model itself. The long-wavelength effective action allows an approximate calculation of the quantities that characterize the superconducting state, such as the mass gap $\Delta(0)$, correlation length $\xi$, London penetration depth $\lambda$, and the critical temperature $T_{c}$, in terms of the basic parameters of the microscopic model: $t, J,+\delta$, and $a .^{18}$

Because of its gauge-field nature, it turns out that the strength of the long-range interhole attraction decreases rapidly as the temperature of the system is raised. This is in stark contrast with BCS theory, where the attractive potential arising from phonon exchange is almost temperature independent. A corresponding analysis of the gap equation shows that this effect reduces the critical temperature in units of the zero-temperature mass gap well below the BCS value. An approximate calculation of the characteristic ratio $2 \Delta(0) / k T_{c}$ yields the value $9.7 .^{10}$ The temperature dependence of the interhole potential depends crucially on the finiteness of the one-loop vacuum polarization diagram and thus is special to gaugeinvariant systems with at most two spatial dimensions. Perhaps the two most unusual features of the high- $T_{c}$ materials $\mathrm{Y}-\mathrm{Ba}-\mathrm{Cu}-\mathrm{O}$ and $\mathrm{La}(\mathrm{Sr}) \mathrm{Cu}-\mathrm{O}$ are their quasitwo-dimensional structure and the high values of the characteristic ratio that they exhibit; this effective theory provides a clear link between these two features.

The physical relevance of any simple model of interacting holes and spins is limited by the recent experimental evidence that up to $70 \%$ of the charge carriers in the $\mathrm{CuO}$ layers of $\mathrm{LA}(\mathrm{Sr}) \mathrm{Cu}-\mathrm{O}$ and $\mathrm{Y}-\mathrm{Ba}-\mathrm{Cu}-\mathrm{O}$ are holes in the oxygen bonds rather than at the copper sites that carry the spins. ${ }^{19}$ The problem of constructing a realistic model involving both types of holes is a formidable one. However, it is certainly possible that all mobile holes are coupled to some extent to the spins and participate in superconductivity due to the mechanism suggested above. The assumption adopted here of a Fermi surface for holes concentrated around isolated points, which yields mass- 
less Dirac fermions in the continuum limit, applies only to the holes at copper sites in physical systems and the introduction of oxygen holes would significantly modify this. Hence the simplified model considered here is not necessarily in contradiction with the results of photoemission experiments, which indicate a complex extended Fermi surface. ${ }^{20}$

${ }^{1}$ G. Bednorz and A. K. Muller, Z. Phys. B 64, 189 (1986).

${ }^{2}$ F. D. M. Haldane, Phys. Lett. 93A, 464 (1983); Phys. Rev. Lett. 50, 1153 (1983); I. Affleck, Nucl. Phys. B257, 397 (1985).

3 J. E. Hirsch and S. Tang, Phys. Rev. Lett. 62, 591 (1989).

${ }^{4}$ R. Shankar, Phys. Rev. Lett. 63, 203 (1989); Nucl. Phys. B330, 433 (1990).

${ }^{5}$ N. Dorey and N. E. Mavromatos, Phys. Lett. B 250, 107 (1990).

${ }^{6}$ N. Dorey and N. E. Mavromatos, Phys. Lett. B 257, 359 (1991).

${ }^{7}$ N. D. Mermin and H. Wagner, Phys. Rev. Lett. 17, 1133 (1966).

${ }^{8}$ S. H. Park, B. Rosenstein, and B. J. Warr (unpublished).

${ }^{9}$ S. Coleman, Commun. Math. Phys. 31, 259 (1973).

${ }^{10}$ N. Dorey and N. E. Mavromatos, Phys. Lett. B (to be published).

${ }^{11}$ I. Ichinose and T. Matsui (unpublished).

${ }^{12}$ B. Rosenstein, B. Warr, and S. H. Park, Phys. Rev. Lett. 62, 1433 (1989).

\section{ACKNOWLEDGMENTS}

The authors would like to thank Brian Pendleton for useful discussions. N.D. acknowledges financial support from the States of Guernsey Education Council. N.E.M. would like to thank the ISI foundation (Torino, Italy) for its hospitality and financial support.

${ }^{13}$ L. B. Iofffe and P. B. Wiegmann, Phys. Rev. Lett. 65, 653 (1990).

${ }^{14}$ G. Baskaran and P. W. Anderson, Phys. Rev. B 37, 580 (1989); I. J. R. Aitchison and N. E. Mavromatos, ibid. 39, 6544 (1989).

${ }^{15} \mathrm{G}$. Baskaran, E. Tosatti, and L. Yu, Int. J. Mod. Phys. B 1, 555 (1988).

${ }^{16}$ I. Affleck and J. B. Marston, Phys. Rev. B 37, 3774 (1988); 39, 11538 (1989).

${ }^{17}$ G. Semenoff and L. C. R. Wijewardhana, Phys. Rev. Lett. 63, 2633 (1989).

${ }^{18}$ N. Dorey and N. E. Mavromatos (unpublished).

${ }^{19}$ A. R. Bishop et al., Z. Phys. B 76, 17 (1989); M. S. Hybertsen et al., Phys. Rev. B 41, 11068 (1990); J. Dutka and A. Oleś, ibid. 42, 105 (1990).

${ }^{20} \mathrm{~A}$. Oleś (private communication). 